Research Paper

\title{
Downregulation of PSCA promotes gastric cancer proliferation and is related to poor prognosis
}

\author{
Li-pu Xu $u^{1,2,3^{*}}$, Hai-bo Qiu ${ }^{1,2,3^{*}}$, Shu-qiang Yuan ${ }^{1,2,3^{*}}$, Yong-ming Chen ${ }^{1,2,3}$, Zhi-wei Zhou ${ }^{1,2,3}$, Ying-bo \\ Chen $1,2,3^{*} \bowtie$
}

1. State Key Laboratory of Oncology in South China, Sun Yat-sen University Cancer Center, Guangzhou, China

2. Collaborative Innovation Center for Cancer Medicine, Sun Yat-sen University Cancer Center, Guangzhou, China

3. Department of Gastric Surgery, Sun Yat-sen University Cancer Center, Guangzhou, China

*These authors contribute equally to this work.

$\square$ Corresponding author: Ying-bo Chen, Email: chenyb@sysucc.org.cn; Tel: $\pm 86-020-87343123$. Department of Gastric Surgery, Sun Yat-sen University Cancer Center, 651 Dong Feng Road East, Guangzhou 510060, China.

(c) The author(s). This is an open access article distributed under the terms of the Creative Commons Attribution License (https://creativecommons.org/licenses/by/4.0/). See http:/ /ivyspring.com/terms for full terms and conditions.

Received: 2019.01.28; Accepted: 2020.01.02; Published: 2020.02.19

\begin{abstract}
Background: Dysregulation of prostate stem cell antigen (PSCA) has been implicated in human cancers. Studies have reported that PSCA expression is generally high in prostate cancer, which correlates with a worse survival. PSCA is also highly expressed in bladder, ovarian, and pancreatic cancers. However, PSCA is expressed at low levels in gastric, gallbladder and oesophageal cancers. At present, the clinical significance, expression pattern and biological function of PSCA in gastric cancer (GC) are still unclear.

Methods: Previously, we used cDNA microarray as a screening tool to compare GC tissues with its matched normal gastric mucosa tissues (MNGT), and obtained the differentially expressed genes of the two tissue types. PSCA is one of the genes significantly down-regulated in GC tissues. In this study, we detected the expression of PSCA in GC tissues and MNGT by western-blot experiment and immunohistochemical staining (IHC). Then the relationship between the expression pattern of PSCA and the clinicopathological characteristics and survival in GC was analyzed. In order to further study the function of PSCA in GC, lentivirus was used to construct stable cell lines with knockdown and overexpression of PSCA gene. We used AGS and MKN45 cell lines for plasmid transfection. Colony formation assay, MTS and nude mice xenograft model were performed to investigate the effect of PSCA in GC.

Results: Western-blot and IHC assays demonstrated that the expression of PSCA in GC tissues was significantly lower than that in the MNGT. PSCA expression in GC tissues was high in $252(57.5 \%)$ and low in $186(42.5 \%)$ of 438 patients. PSCA expression for MNGT was high in $273(62.3 \%)$ and low in $165(37.7 \%)$ of 438 patients. PSCA expression was significantly associated with $T$ classification $(P=0.024), N$ classification $(P=0.018)$ and TNM stage $(P=0.019)$ using $X^{2}$ test. The relationship between PSCA expression level and patient survival was analysed using Kaplan-Meier analysis and the log-rank test. Low levels of PSCA expression were significantly associated with a poorer OS than high expression levels of $\mathrm{PSCA}(\mathrm{P}=0.011)$. In the COX regression analysis of OS, all 9 variables in the univariate analysis were significantly correlated with OS $(\mathrm{P}<0.05)$, while the variables found to be independently correlated with OS in the multivariate analysis were PSCA expression $(P=0.036)$, age $(P<0.001)$, gender $(P=0.007)$, and TNM stage $(P<0.001)$, respectively. Univariate and multivariate analyses showed that PSCA was an independent prognostic factor for OS in GC. In vitro MTS cell proliferation experiment and clonal formation experiment and in vivo nude mouse subcutaneous tumorigenesis experiment all proved that knockdown of PSCA gene can improve the proliferation ability of GC cells, while in vitro experiment proved that overexpression of PSCA can reduce the proliferation ability of GC cells.It was found that knockdown of PSCA gene can improve the proliferation ability of GC cells both in vitro and in vivo, while overexpression of PSCA can reduce the proliferation ability of GC cells in vitro.

Conclusion: Our study showed that the expression of PSCA gene was decreased in GC, which was related to more advanced pathological stages. And the expression level of PSCA in GC was an independent good prognostic factor. PSCA gene had the function of inhibiting GC proliferation.
\end{abstract}

Key words: $P S C A$, gastric cancer, prognosis, survival, proliferation 


\section{Introduction}

Gastric cancer (GC) is the fifth most common cancer and was the third leading cause of cancer deaths worldwide in 2018. [1] More than 1 million new GC cases were diagnosed in 2018, and more than 783 thousand deaths were attributed to GC.[1] That is, approximately 1 in every 12 deaths was caused by GC globally.[1] To date, surgical resection remains the primary treatment for GC. However, most patients are diagnosed with advanced or stage IV TNM GC.[2] Operation alone has a limited effect on patient survival. Therefore, chemotherapy and targeted therapy are of great importance for GC treatment. To date, targeted drugs applied in GC are limited to ramucirumab and trastuzumab.[3-5] More targeted drugs are required to be exploited and to be used in the clinical treatment of GC.

PSCA gene function is to encode a glycosylphosphatidylinositol-anchored cell membrane glycoprotein, which is related to cell adhesion, proliferation, and survival. PSCA is on chromosome $8 \mathrm{q} 24.3$ with a polymorphism that induced different risks for different cancers.[6] Studies have reported that PSCA expression is generally high in prostate cancer,[7] which correlates with a worse survival. PSCA is also highly expressed in bladder, ovarian, and pancreatic cancers.[8-10] However, PSCA is expressed at low levels in gastric, gallbladder and oesophageal cancers. $[10,11]$ These results indicate that PSCA has different functions in different tissue types. Initially, PSCA was considered to participate in signal transduction, and several studies have shown its involvement in cell growth regulation.[9-11] Subsequently, studies have shown PSCA promotes prostate cancer growth by increasing c-myc expression through the PI3K/AKT signalling pathway [12]. Nevertheless, a study of PSCA in esophageal cancer showed that PSCA plays a tumor suppressor role by promoting the nuclear translocation of RB1CC1.[13] Recently, a genome-wide Association Study (GWAS) in Japan found that PSCA gene loci rs2294008, rs2976392 and other variants were associated with the incidence of gastric adenocarcinoma.[14] However, the role of PSCA in GC is still unknown, and the function of PSCA in GC has not been fully explored.

Previously, we used cDNA microarrays as a screening tool; we compared GC tissues with their MNGT and obtained genes with differential expression in both tissue types. PSCA was one of the significantly downregulated genes in GC tissues. Therefore, in this study, we examined PSCA expression in GC tissues and MNGT, compared the clinicopathologic and prognostic differences between the high and low expression levels in PSCA patients, and investigated the function of PSCA in GC, laying the foundation for the next step to clarify the mechanism of PSCA function in GC.

\section{Materials and Methods}

\section{Patients and tissue samples}

All 438 pairs of paraffin-embedded GC tissues and their MNGT were obtained from surgical resection at Sun Yat-sen University Cancer Center and then made into tissue microarrays (TMAs). All the patients involved in this study signed informed consent agreements. The use of paraffin-embedded tissues in this study was approved by the Ethics Committee of Sun Yat-sen University Cancer Center. The patients were selected continuously retrospecttively from operation time during March 2010 to November 2013 in our department, included the conditions as follows:1) no history of neoadjuvant therapy, 2) underwent gastric resection, whatever operation kind (palliative or radical, partial or total gastric resection, D1,D2 or D3 lymphadenectomy, R0 or $\mathrm{R} 1$ resection), 3) no history of malignant tumour other than GC, 4) postoperative pathology confirmed gastric adenocarcinoma or squamous carcinoma. Exclusive criteria were: 1) no gastrectomy, and 2) R2 resection. Follow-ups were conducted by the Department of Follow-up in our hospital every 3 months after surgery for the first 2 years, every 6 months from the third to fifth years after surgery, and then once a year until patient death. The TNM stage was measured using The American Joint Committee on Cancer (AJCC, $8^{\text {th }}$ edition).

\section{Immunohistochemistry}

Immunohistochemistry was performed on the abovementioned paraffin-embedded tissues. The tissues were fixed, paraffin-embedded and cut into 5 $\mu \mathrm{m}$ thick slices. IHC staining was performed according to the standard IHC protocol. First of all, one of the TMA slides were placed on the microscope which was connected to the computer. On the computer side set the diameter size of a circular tissue point in the TMA slide, adjusted the appropriate focal length. Next, the microscope took several photos for each tissue point of the TMA slide, then the computer jointed the photos together into a complete picture of the TMA slide and marked each tissue point of the TMA slide. The TMA IHC images were photographed by a TMA scanning system (Vectra 2.0.4982.22572, Perkin Elmer, Inc.). Then the computer analyzed each tissue point of the TMA slide by tissue scanning analysis system software provided with the instrument (Perkin Elmer, Inc.). All sells in a marked tissue point were evaluated by the computer software for staining intensity and then the percentages of each intensity grade were calculated. The staining intensity 
was defined as follows: 0 (negative), 1 (weak), 2 (moderate), and 3 (strong). The immunoreactive score (IRS) was multiplied by the grade of positive cell percentages and the staining intensity of the tissue. The final score of one TMA tissue was added by each staining intensity multiplied by its own percentage * 100. For example, if a TMA tissue staining intensity was $0(18.92 \%), 1+(80.61 \%), 2+(0.47 \%)$ and $3+$ $(0.00 \%)$, then the final score was $(0 * 18.92 \%+1 * 80.61 \%$ $+2 * 0.47 \%+3 * 0.00 \%) * 100=82$. The results of all TMA tissues ranged from 2 to 198 . We defined the level of PSCA as low (score $<78$ ) and high (score $\geq 78$ ) to make sure enough number of cases for both groups.

\section{Cell lines and cell culture}

MKN45 and AGS cells were acquired from the Shanghai Cell Bank of the Chinese Academy of Sciences (Shanghai, China). MKN45 cells were cultured in RMPI-1640 media (Gibco) supplemented with $10 \%$ foetal bovine serum (Gibco). AGS cells were cultured in F-12K media (Gibco) supplemented with $10 \%$ foetal bovine serum (Gibco). MKN45 and AGS cells grew in standard cell culture conditions $\left(37^{\circ} \mathrm{C}\right.$, $5 \% \mathrm{CO}_{2}$, and $95 \%$ humidity).

\section{Antibodies}

Anti-PSCA rabbit polyclonal antibody was purchased from Abcam (ab64919, Abcam, Inc., Cambridge, MA). Anti- $\beta$-actin mouse monoclonal antibody, goat anti-rabbit conjugated to horseradish peroxidase secondary antibody, and goat anti-mouse conjugated to horseradish peroxidase secondary antibody were purchased from Cell Signaling Technology (CST).

\section{Western blot analysis}

Total protein was extracted from GC tumour tissues and MNGT using lysis buffer. Tissues were obtained from surgery resection and confirmed by pathological diagnosis. Total protein was measured by a BCA kit and then made into protein samples. Protein samples were separated by sodium dodecyl sulphate polyacrylamide gel electrophoresis (SDSPAGE) using a Bis-Tris gel and were transferred to PVDF membranes by wet transfer. The acrylamide concentration was $15 \%$. The gel was run at $80 \mathrm{~V}$ for 30 min and $120 \mathrm{~V}$ for $65 \mathrm{~min}$. Then, the gel was transferred to PVDF membranes at 0.25 A for $90 \mathrm{~min}$. The PVDF membranes were blocked in 5\% skim milk at $4{ }^{\circ} \mathrm{C}$ overnight and then incubated in anti-PSCA rabbit polyclonal antibody (1:1000 dilution) and anti- $\beta$-actin mouse monoclonal antibody (1:1000 dilution) at $4{ }^{\circ} \mathrm{C}$ overnight. The PVDF membranes were incubated in goat anti-rabbit conjugated to horseradish peroxidase secondary antibody (1:3000 dilution) and goat anti-mouse conjugated to horseradish peroxidase secondary antibody (1:5000 dilution) for $1 \mathrm{~h}$. The membranes were visualized via ECL reagents (Bio-Rad, USA) and measured by the Bio-Rad instrument.

\section{MTS assay}

The cell proliferation rate was measured by a 3-(4,5-dimethylthiazol-2-yl)-5-(3-carboxymethoxyphe nyl)-2-(4-sulfophenyl)-2H-tetrazolium (MTS) assay (Promega Corporation, Fitchburg, WI). According to the instructions, 2000 cells were plated in wells of a 96-well plate. A total of 6 plates were used for 1-6 days. MTS $(10 \mu \mathrm{l})$ was added to the wells and then incubated in a cell incubator for $4 \mathrm{~h}$. The plates were measured at $490 \mathrm{~nm}$ wavelength in the machine.

\section{Colony formation assay}

MKN45, MKN45-SH, AGS and AGS-NM cells were seeded in 6-well plates at 500 cells per well and cultured for 10 or 14 days. Cells were fixed with $4 \%$ paraformaldehyde solution, stained with $0.1 \%$ crystal violet, and counted under an inverted microscope.

\section{Slow virus and transfection}

Plasmids, including knockdown or overexpresssion sequences, were designed and packaged as lentiviruses by GenePharma. The knockdown sequences were 5'-GCTGTGACACCGACTTGTGCA$3^{\prime}$. Transfection steps were performed according to GenePharma protocols. Selection was performed using puromycin for stable transfection for 14 days.

\section{Nude mice xenograft}

The Research Animal Resource Center of Sun Yat-sen University approved the use of laboratory animals in this study. BALB/c athymic nude mice (4 weeks old) were purchased from Beijing Vital River Laboratory Animal Technology Co., Ltd. (Beijing, China). The experiments and procedures on animals conformed to the institutional animal care guidelines. A xenograft model was used. The mice were injected with $5 \times 10^{6}$ MKN45 cells or MKN45-shPSCA cells in the right subcutaneous armpit. Tumour size was measured twice a week. Mice were sacrificed after the size of the tumour exceeded $1000 \mathrm{~mm}^{3}$.

\section{Results}

\section{PSCA expression in GC patients and its relationship to clinicopathological characteristics}

To investigate the relationship between PSCA expression and the clinicopathological features of GC patients, IHC was performed to evaluate the PSCA expression pattern of 438 pairs of paraffin-embedded GC tissues and their MNGT in TMA. The PSCA 
antibody specifically recognized PSCA in the cellular membrane and cytoplasm. PSCA expression in GC tissues was high in 252 (57.5\%) and low in 186 (42.5\%) of 438 patients. PSCA expression for MNGT was high in $273(62.3 \%)$ and low in $165(37.7 \%)$ of 438 patients. The difference in PSCA expression indicates that PSCA protein levels were lower in tumour tissues than in MNGT tissues (Table 1). Western blot analysis further confirmed these results (Figure A_a1, Figure A_a2). The general characteristics of patients is shown in Table 2. Typical PSCA staining intensity images (negative, weak, moderate and strong) from TMA tissues are presented in Figure A_b. As shown in Table 3, PSCA expression was significantly associated with $\mathrm{T}$ classification $(\mathrm{P}=0.024), \mathrm{N}$ classification $(\mathrm{P}=0.018)$ and TNM stage $(\mathrm{P}=0.019)$. The relationship between PSCA expression level and patient survival was analysed using Kaplan-Meier analysis (Figure B) and the log-rank test. Low levels of PSCA expression were significantly associated with a poorer OS than high expression levels of PSCA $(\mathrm{P}=0.011)$. These results suggest that PSCA is related to GC tumourigenesis. The univariate and multivariate analysis of COX regression for overall survival (Table 4) suggested that PSCA expression was an independent prognostic factor for gastric cancer $(\mathrm{P}=0.036)$.

\section{PSCA decelerates GC cell proliferation in vitro}

First, the PSCA expression level in various GC cell lines was assessed. The highest expression level of PSCA was in MKN45 cells, and the lowest expression level is AGS cells (Figure C_a). Therefore, MKN45 and AGS cells were selected to evaluate the function of PSCA in GC cells. Lentiviral vectors expressing PSCA and PSCA shRNA sequences were used to establish stable cell lines. MKN45-shPSCA and AGS-PSCA were then constructed by lentivector transduction. The WB assay confirmed that the silencing operation in MKN45 cells and the overexpression operation in AGS cells succeeded (Figure C_b, Figure C_c).

Figure A_a1

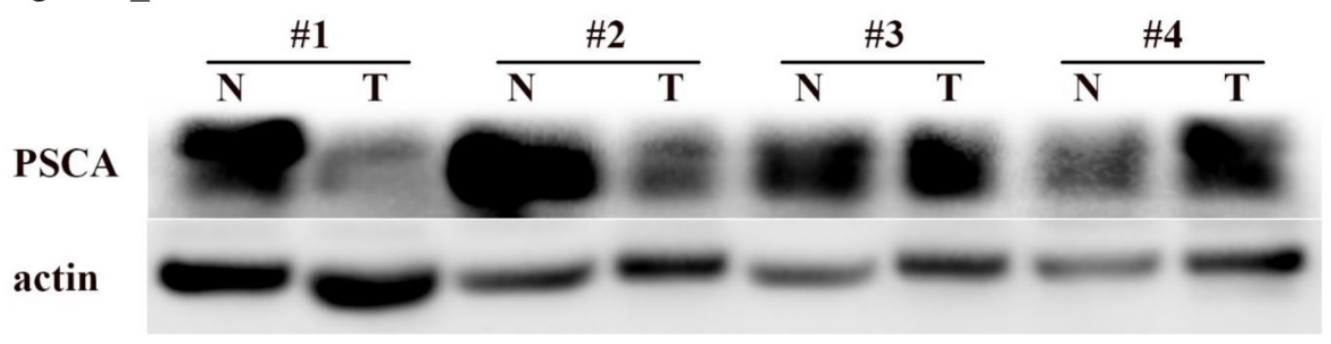

Figure A_a2

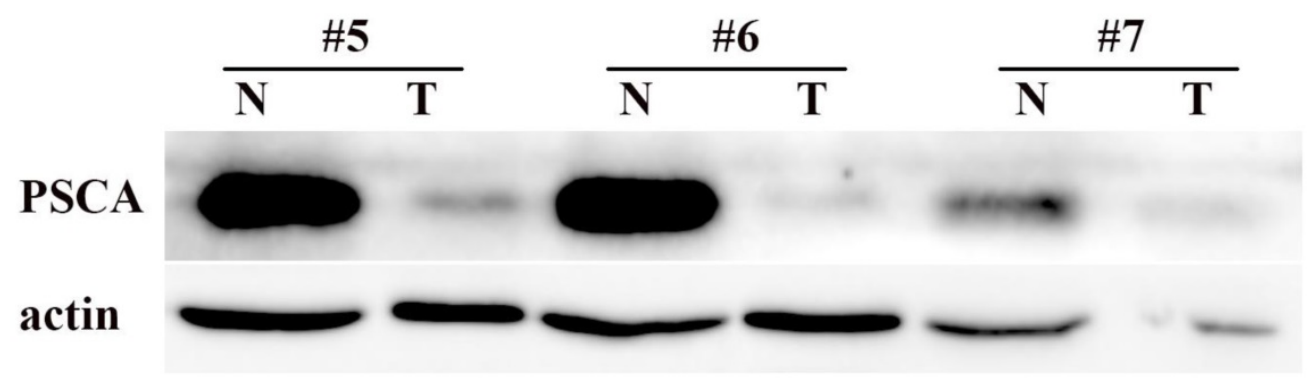

Figure A_b
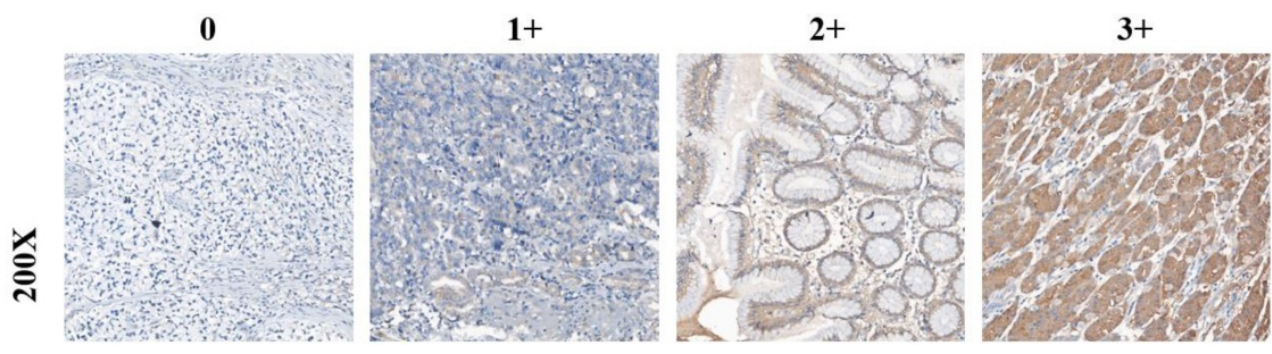

Figure A. PSCA is lowly expressed in GC tissues. (a) Western blot of PSCA expression in 7 pairs of representative GC tissues (T) with MNGT. (b) Immunohistochemical staining of PSCA in four pairs of representative GC tissues (T) with MNGT. 


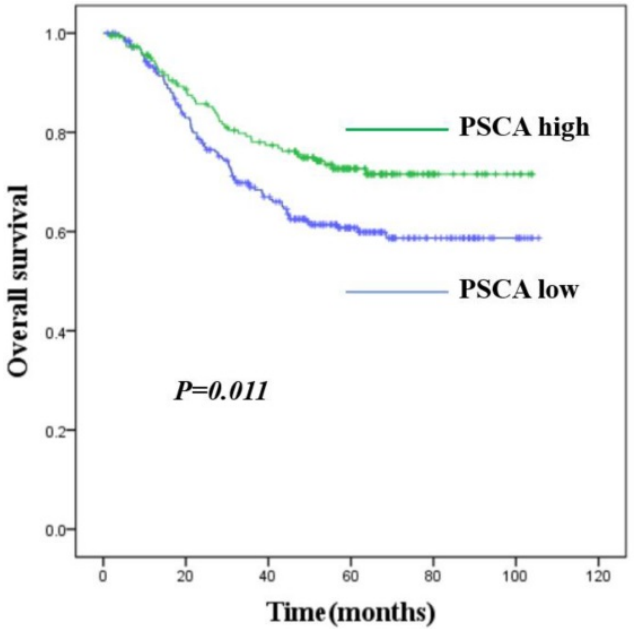

Figure B. Kaplan-Meier survival curves for GC patients with high PSCA expression versus low PSCA expression. The overall survival of patients of GC with high or low PSCA expression $(\mathrm{P}=0.011)$.

Table 1. Difference of PSCA expression between GC and MNGT using IHC staining.

\begin{tabular}{lllll}
\hline Tissue type & \multicolumn{2}{l}{ PSCA expression } & Total & P value \\
\cline { 2 - 3 } & low & high & & \\
\hline MNGT & $165(37.7 \%)$ & $273(62.3 \%)$ & 438 & $<0.001$ \\
$\mathrm{~T}$ & $252(57.5 \%)$ & $186(42.5 \%)$ & 438 & \\
\hline
\end{tabular}

Table 2. Clinicopathological characteristics and PSCA expression in 438 GC patients

\begin{tabular}{ll}
\hline Characteristics & Number of cases (\%) \\
\hline Age (years) & $307(70.1)$ \\
$<65$ & $131(29.9)$ \\
$\geq 65$ & $298(68.0)$ \\
Gender & $140(32.0)$ \\
Male & \\
Female & $280(63.9)$ \\
Tumour size(cm) & $158(36.1)$ \\
$<5$ & \\
$\geq 5$ & $12(2.7)$ \\
Histologic grade & $50(11.4)$ \\
Well & $376(85.9)$ \\
Moderate & \\
Poor & $67(15.3)$ \\
pT classification & $50(11.4)$ \\
T1 & $153(34.9)$ \\
T2 & $168(38.4)$ \\
T3 & \\
T4 & $140(32.0)$ \\
pN classification & $74(16.9)$ \\
N0 & $77(17.6)$ \\
N1 & $147(33.5)$ \\
N2 & \\
N3 & $406(92.7)$ \\
pM classification & $32(7.3)$ \\
M0 & \\
M1 & $79(18.0)$ \\
pTNM stage & $128(29.2)$ \\
I & $199(45.4)$ \\
II & $32(7.4)$ \\
III & \\
IV & $300(68.5)$ \\
Vital status & $138(31.5)$ \\
Alive & \\
Death & $252(57.5)$ \\
Expression of PSCA & $186(42.5)$ \\
Low expression & \\
High expression & \\
\hline
\end{tabular}

Table 3. Correlation between PSCA expression and clinicopathological variables in 438 GC cases

\begin{tabular}{|c|c|c|c|c|}
\hline \multirow[t]{2}{*}{ Characteristics } & & \multicolumn{2}{|c|}{ PSCA expression } & \multirow[t]{2}{*}{$P$ value } \\
\hline & & Low & High & \\
\hline \multirow[t]{2}{*}{ Age (years) } & $<65$ & 176 & 131 & 0.894 \\
\hline & $\geq 65$ & 76 & 55 & \\
\hline \multirow[t]{2}{*}{ Gender } & Male & 174 & 124 & 0.597 \\
\hline & Female & 78 & 62 & \\
\hline \multirow[t]{2}{*}{ Tumour size $(\mathrm{cm})$} & $<5$ & 160 & 120 & 0.825 \\
\hline & $\geq 5$ & 92 & 66 & \\
\hline \multirow[t]{3}{*}{ Histologic grade } & Well & 6 & 6 & 0.736 \\
\hline & Moderate & 27 & 23 & \\
\hline & Poor & 219 & 157 & \\
\hline \multirow[t]{3}{*}{ T classification } & $\mathrm{T} 1$ & 33 & 34 & $0.024^{*}$ \\
\hline & $\mathrm{T} 2+\mathrm{T} 3$ & 109 & 94 & \\
\hline & $\mathrm{T} 4$ & 110 & 58 & \\
\hline \multirow[t]{5}{*}{$\mathrm{N}$ classification } & No & 69 & 71 & $0.018^{*}$ \\
\hline & N1 & 44 & 30 & \\
\hline & $\mathrm{N} 2$ & 43 & 34 & \\
\hline & N3a & 81 & 49 & \\
\hline & $\mathrm{N} 3 \mathrm{~b}$ & 15 & 2 & \\
\hline \multirow[t]{2}{*}{ M classification } & M0 & 232 & 174 & 0.555 \\
\hline & M1 & 20 & 12 & \\
\hline \multirow[t]{2}{*}{ pTNM stage } & I+II & 107 & 100 & $0.019^{*}$ \\
\hline & III+IV & 145 & 86 & \\
\hline
\end{tabular}

${ }^{*}$ P-value $<0.05$

Table 4. Univariate and multivariate Cox proportional analysis with overall survival.

\begin{tabular}{|c|c|c|c|c|}
\hline \multirow[b]{2}{*}{ Parameters } & \multicolumn{2}{|l|}{ Univariate analysis } & \multicolumn{2}{|c|}{ Multivariate analysis } \\
\hline & $\mathrm{HR}^{\mathrm{a}}\left(95 \% \mathrm{CI}^{\mathrm{b}}\right)$ & $\mathbf{P}$ & HR $(95 \%$ CI) & $\mathbf{P}$ \\
\hline PSCA expression & $0.637(0.448-0.906)$ & $0.012^{*}$ & $0.683(0.479-0.975)$ & $0.036^{*}$ \\
\hline \multicolumn{5}{|l|}{ Low } \\
\hline \multicolumn{5}{|l|}{ High } \\
\hline Age & $1.757(1.250-2.471)$ & $0.001^{* *}$ & $2.007(1.422-2.834)$ & $<0.001^{* *}$ \\
\hline \multicolumn{5}{|l|}{$<65$} \\
\hline \multicolumn{5}{|l|}{$\geq 65$} \\
\hline Sex & $1.533(1.091-2.153)$ & $0.014^{*}$ & $1.609(1.141-2.269)$ & $0.007^{*}$ \\
\hline \multicolumn{5}{|l|}{ Male } \\
\hline \multicolumn{5}{|l|}{ Female } \\
\hline Tumour size $(\mathrm{cm})$ & $1.802(1.288-2.521)$ & $0.001^{* *}$ & & 0.337 \\
\hline \multicolumn{5}{|l|}{$<5$} \\
\hline \multicolumn{5}{|l|}{$\geq 5$} \\
\hline Histologic Grade & $1.820(1.028-3.223)$ & $0.040^{*}$ & & 0.461 \\
\hline \multicolumn{5}{|l|}{ Well+moderate } \\
\hline \multicolumn{5}{|l|}{ Poor } \\
\hline TNM Stage & $5.985(3.874-9.246)$ & $<0.001^{* * *}$ & $6.053(3.912-9.365)$ & $<0.001^{* *}$ \\
\hline \multicolumn{5}{|l|}{ I-II } \\
\hline \multicolumn{5}{|l|}{ III-IV } \\
\hline T classification & $2.321(1.842-2.926)$ & $<0.001^{* * *}$ & & \\
\hline \multicolumn{5}{|l|}{$\mathrm{T} 1$} \\
\hline \multicolumn{5}{|l|}{ T2 } \\
\hline \multicolumn{5}{|l|}{$\mathrm{T} 3$} \\
\hline \multicolumn{5}{|l|}{$\mathrm{T} 4$} \\
\hline $\mathbf{N}$ classification & $1.969(1.688-2.296)$ & $<0.001^{* * *}$ & & \\
\hline \multicolumn{5}{|l|}{ No } \\
\hline \multicolumn{5}{|l|}{ N1 } \\
\hline \multicolumn{5}{|l|}{ N2 } \\
\hline \multicolumn{5}{|l|}{ N3a } \\
\hline \multicolumn{5}{|l|}{ N3b } \\
\hline M classification & $5.273(3.251-8.550)$ & $<0.001^{* * *}$ & & \\
\hline M0 & & & & \\
\hline M1 & & & & \\
\hline
\end{tabular}

aHR, hazard ratio; bCI confidence interval. *P-value $<0.05 * * * \mathrm{P}$-value $<0.01$

MTS assays were performed to evaluate cell proliferation. Downregulated PSCA levels in MKN45 cells significantly increased cell growth compared with MKN45 cells (Figure D_a, P=0.001). On the other hand, upregulated PSCA levels in AGS cells 
decreased cell growth compared to control cells (Figure D_a, P<0.001).

As shown in Figure D_b, the colony formation assay was in accordance with the observation. The number of colonies for MKN45-shPSCA was significantly higher than the numbers of the MKN45 control cells, and the AGS-PSCA cells formed more colonies than those of the AGS cells.

\section{PSCA decelerates GC cell proliferation in vivo}

To further investigate the observation that PSCA suppresses GC cell proliferation. Nude mice xenograft tumour model assays were performed. Male nude mice were divided into two groups with five animals in each group. A total of $5 \times 10^{6}$ of MKN45 or MKN45-shPSCA cells per mouse were injected into the right subcutaneous axillary in each group. As shown in Figure E_b, the tumour volumes were significantly higher in the MKN45-shPSCA group than in the MKN45 group at every specific measuring time. The mice were sacrificed using cervical dislocation after 4 weeks when the largest tumour volume reached $1000 \mathrm{~mm}^{3}$. The tumour was excised from each mouse, and the weight of the tumour was measured. Again, the tumour weights in the MKN45-shPSCA group were significantly greater than those in the MKN45 group (Figure E_a, P=0.037).

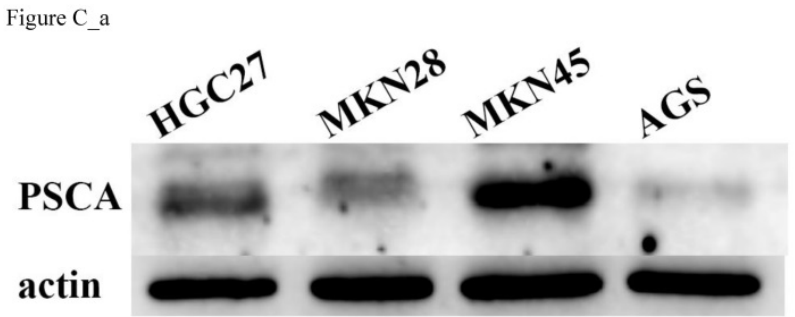

Figure C_b

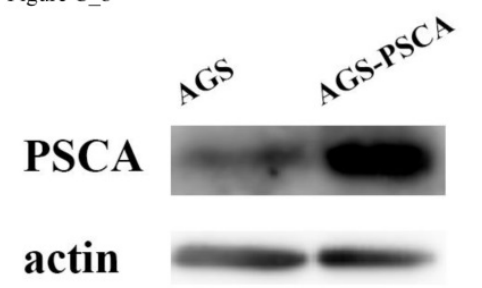

Figure C_c

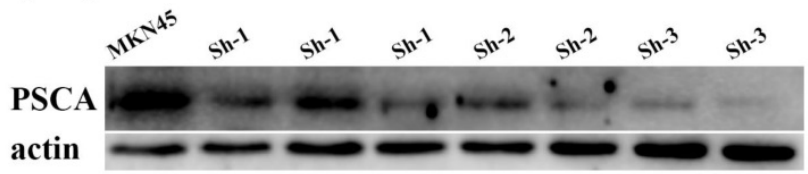

Figure C. PSCA expression in cell lines and stable cell lines construction. (a) PSCA expression is lowest in AGS, and is highest in MKN45. (b) Overexpression of PSCA stable cell line AGS-PSCA was constructed. (c) Downregulation of PSCA stable cell line MKN45-shPSCA was constructed using the last cell line (sh-3).
Figure D a

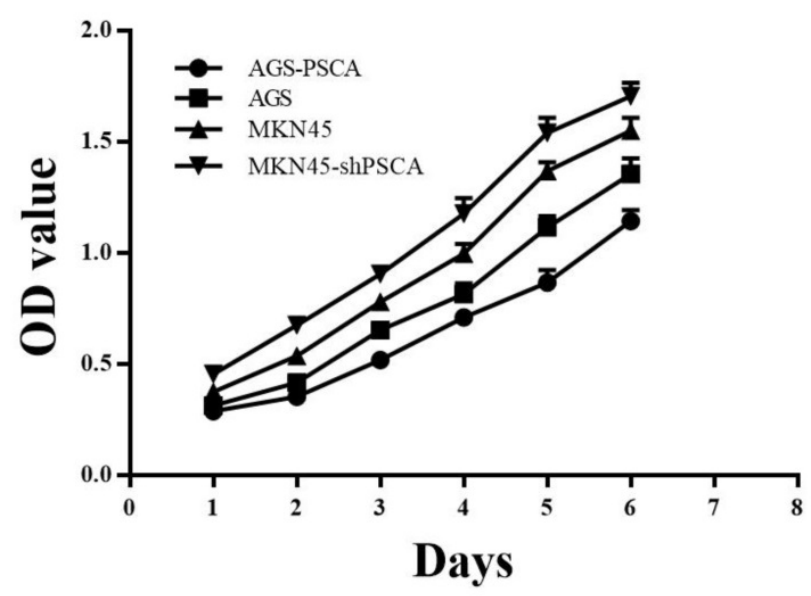

Figure D_b1

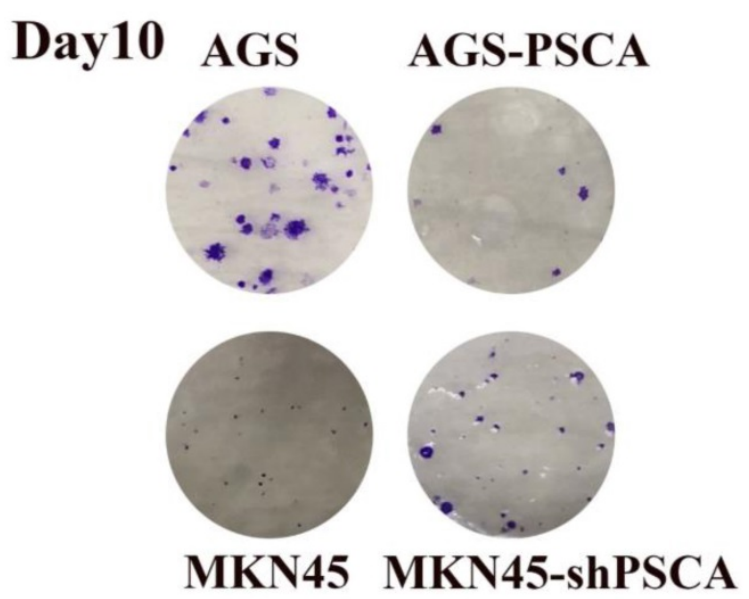

Figure D_b2

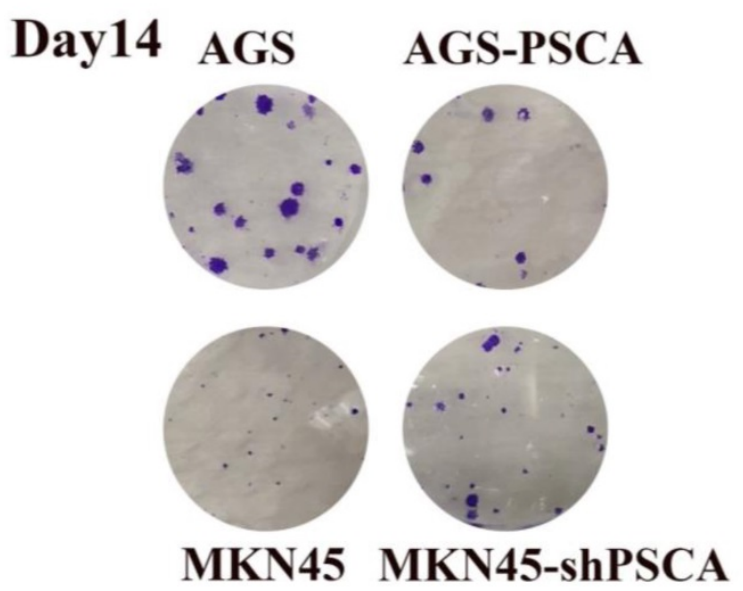

Figure D. Effects of PSCA on the cell proliferation in vitro. (a) MTS assay indicated that knockdown of PSCA in MKN45-shPSCA cells increased the cell proliferation, overexpression of PSCA in AGS-PSCA cells decreased the cell proliferation. (b) Colony formation assay indicated that knockdown of PSCA in MKN45-shPSCA cells result to more and bigger colonies, overexpression of PSCA in AGS-PSCA cells result to less and smaller colonies at day10 and 14. 


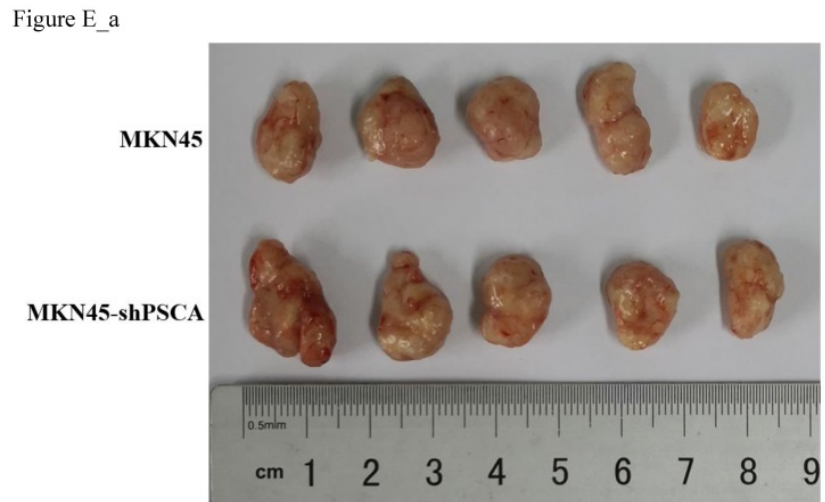

Figure E b

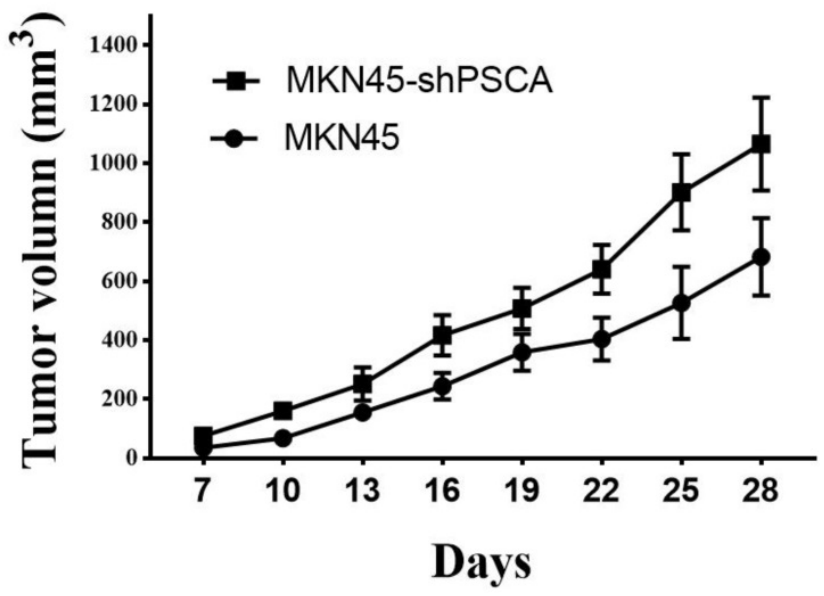

Figure E. Effects of PSCA on the cell growth in vivo. (a) Tumour excised from nude mice after 4 weeks growth and then weighted. (b) Tumour volume were measured using the formula $0.5 \times$ (length $) \times(\text { width })^{2}$.

\section{Discussion}

In our previous cDNA microarray screening, we found that PSCA was significantly expressed at low levels in GC. To further investigate the role of PSCA in GC, we performed western blot and IHC assays. A total of 438 pairs of GC tissues with its MNGT were selected to compare the expression difference of PSCA, and found that PSCA gene expression was significantly lower in GC tissues which was consistent with the study of Sakamoto et al.[11] and the sequencing results in our department. A GWAS study predicted that low PSCA expression might be associated with more advanced clinical staging and poor prognosis.[15] Our study found that low expression of PSCA was associated with higher $\mathrm{pT}$ staging $(\mathrm{P}=0.024), \mathrm{pN}$ staging $(\mathrm{P}=0.018)$, and $\mathrm{pTNM}$ staging $(\mathrm{P}=0.019)$, especially in 15 of the 17 patients with pN3b. Kaplan-meier survival analysis showed that low PSCA expression was associated with poor prognosis $\quad(\mathrm{P}=0.011)$, while univariate and multivariate analysis showed that PSCA expression was an independent prognostic factor in GC $(\mathrm{P}=0.036)$.
PSCA encodes a GPI-anchored cell membrane protein that belongs to the Thy-1/Ly-6 family.[6] The protein was predicted to be in the function of cell adhesion, proliferation, and survival.[16-18] Several studies have suggested that PSCA may play a role in signal transduction and cell growth regulation.[19-21] Previous research have found that PSCA is highly expressed in prostate cancer, bladder cancer, renal cell cancer, pancreatic cancer, ovarian cancer and other cancer, but low in esophageal cancer and GC.[15, 22, 23] Later studies have shown that PSCA promotes prostate cancer by increasing the expression of c-myc through the PI3K/AKT signalling pathway.[12] A study of PSCA in esophageal cancer has shown that PSCA plays a tumour suppressor role by promoting the nuclear translocation of RB1CC1 [13].

Our study indicated that PSCA suppressed cell proliferation in GC, and knockdown of PSCA gene promotes cell proliferation both in vitro and in vivo. PSCA acts a tumour suppressor role in GC, similar to the function of PSCA in esophageal cancer, but contrary to the function of PSCA in prostate cancer and nasopharyngeal cancer.[6] All these findings demonstrated that PSCA plays different biological functions in different tissue types.[24] PSCA functions in tumour growth are tissue-dependent.

It needs to be noted that the specific mechanism of PSCA inhibiting the proliferation of GC has not been fully explored in this study. So far, the mechanism of PSCA in GC is still unknown, PSCA plays a tumour suppressor role in GC and esophageal cancer. Whether PSCA has similar results in other gastrointestinal tumour, such as colon cancer, remains to be studied. In addition, why PSCA has different functions in different tissue types has not been fully explored at present. More studies on the expression patterns, functions, outcomes and biological mechanisms of PSCA in different tissues and cell types are needed.

\section{Acknowledgements}

This work was supported by research grants from the National Natural Science Foundation of China (No. 81602061).

\section{Competing Interests}

The authors have declared that no competing interest exists.

\section{References}

1. Bray F, Ferlay J, Soerjomataram I, Siegel RL, Torre LA, Jemal A. Global cancer statistics 2018: GLOBOCAN estimates of incidence and mortality worldwide for 36 cancers in 185 countries. CA: a cancer journal for clinicians. 2018; 68: 394-424

2. Cunningham D. Gastric cancer--the recognition of a chemosensitive tumour. British journal of cancer. 1988; 58: 695-9.

3. Bang YJ, Van Cutsem E, Feyereislova A, Chung HC, Shen L, Sawaki A, et al. Trastuzumab in combination with chemotherapy versus chemotherapy alone 
for treatment of HER2-positive advanced gastric or gastro-oesophageal junction cancer (ToGA): a phase 3, open-label, randomised controlled trial. Lancet. 2010; 376: 687-97.

4. Fuchs CS, Tomasek J, Yong CJ, Dumitru F, Passalacqua R, Goswami C, et al. Ramucirumab monotherapy for previously treated advanced gastric or gastro-oesophageal junction adenocarcinoma (REGARD): an international, randomised, multicentre, placebo-controlled, phase 3 trial. Lancet. 2014; 383: 31-9.

5. Wilke H, Muro K, Van Cutsem E, Oh SC, Bodoky G, Shimada Y, et al. Ramucirumab plus paclitaxel versus placebo plus paclitaxel in patients with previously treated advanced gastric or gastro-oesophageal junction adenocarcinoma (RAINBOW): a double-blind, randomised phase 3 trial. The Lancet Oncology. 2014; 15: 1224-35.

6. Reiter RE, Gu Z, Watabe T, Thomas G, Szigeti K, Davis E, et al. Prostate stem cell antigen: a cell surface marker overexpressed in prostate cancer. Proceedings of the National Academy of Sciences of the United States of America. 1998; 95: 1735-40.

7. Gu Z, Thomas G, Yamashiro J, Shintaku IP, Dorey F, Raitano A, et al. Prostate stem cell antigen (PSCA) expression increases with high gleason score, advanced stage and bone metastasis in prostate cancer. Oncogene. 2000; 19: 1288-96

8. Argani P, Rosty C, Reiter RE, Wilentz RE, Murugesan SR, Leach SD, et al. Discovery of new markers of cancer through serial analysis of gene expression: prostate stem cell antigen is overexpressed in pancreatic adenocarcinoma. Cancer research. 2001; 61: 4320-4.

9. Cao D, Ji H, Ronnett BM. Expression of mesothelin, fascin, and prostate stem cell antigen in primary ovarian mucinous tumors and their utility in differentiating primary ovarian mucinous tumors from metastatic pancreatic mucinous carcinomas in the ovary. International journal of gynecological pathology : official journal of the International Society of Gynecological Pathologists. 2005; $24: 67-72$

10. Bahrenberg G, Brauers A, Joost HG, Jakse G. Reduced expression of PSCA, a member of the LY-6 family of cell surface antigens, in bladder, esophagus, and stomach tumors. Biochemical and biophysical research communications. 2000; 275: 783-8.

11. Study Group of Millennium Genome Project for C, Sakamoto H, Yoshimura K, Saeki N, Katai H, Shimoda T, et al. Genetic variation in PSCA is associated with susceptibility to diffuse-type gastric cancer. Nature genetics. 2008; 40: $730-40$.

12. Li E, Liu L, Li F, Luo L, Zhao S, Wang J, et al. PSCA promotes prostate cancer proliferation and cell-cycle progression by up-regulating c-Myc. The Prostate. 2017; 77: 1563-72.

13. Zhang LY, Wu JL, Qiu HB, Dong SS, Zhu YH, Lee VH, et al. PSCA acts as a tumor suppressor by facilitating the nuclear translocation of RB1CC1 in esophageal squamous cell carcinoma. Carcinogenesis. 2016; 37: 320-32.

14. Hishida A, Ugai T, Fujii R, Nakatochi M, Wu MC, Ito H, et al. GWAS analysis reveals a significant contribution of PSCA to the risk of Heliobacter pylori-induced gastric atrophy. Carcinogenesis. 2019; 40: 661-668.

15. Shi Y, Hu Z, Wu C, Dai J, Li H, Dong J, et al. A genome-wide association study identifies new susceptibility loci for non-cardia gastric cancer at 3q13.31 and 5p13.1. Nature genetics. 2011; 43: 1215-8.

16. Saeki N, Gu J, Yoshida T, Wu X. Prostate stem cell antigen: a Jekyll and Hyde molecule? Clinical cancer research : an official journal of the American Association for Cancer Research. 2010; 16: 3533-8.

17. Kawaguchi $\mathrm{T}$, Sho $\mathrm{M}$, Tojo $\mathrm{T}$, Yamato $\mathrm{I}$, Nomi $\mathrm{T}$, Hotta $\mathrm{K}$, et al. Clinical significance of prostate stem cell antigen expression in non-small cell lung cancer. Japanese journal of clinical oncology. 2010; 40: 319-26.

18. Marra E, Uva P, Viti V, Simonelli V, Dogliotti E, De Rinaldis E, et al. Growth delay of human bladder cancer cells by Prostate Stem Cell Antigen downregulation is associated with activation of immune signaling pathways. BMC cancer. 2010; 10: 129.

19. Tran CP, Lin C, Yamashiro J, Reiter RE. Prostate stem cell antigen is a marker of late intermediate prostate epithelial cells. Molecular cancer research : MCR. 2002; 1: 113-21.

20. Saffran $D C$, Raitano $A B$, Hubert RS, Witte $O N$, Reiter RE, Jakobovits A Anti-PSCA mAbs inhibit tumor growth and metastasis formation and prolong the survival of mice bearing human prostate cancer xenografts. Proceedings of the National Academy of Sciences of the United States of America. 2001; 98: 2658-63.

21. Gu Z, Yamashiro J, Kono E, Reiter RE. Anti-prostate stem cell antigen monoclonal antibody 1G8 induces cell death in vitro and inhibits tumor growth in vivo via a Fc-independent mechanism. Cancer research. 2005; 65: 9495-500

22. Cui H, Tang M, Zhang M, Liu S, Chen S, Zeng Z, et al. Variants in the PSCA gene associated with risk of cancer and nonneoplastic diseases: systematic research synopsis, meta-analysis and epidemiological evidence. Carcinogenesis. 2019; 40: 70-83

23. Tanikawa C, Urabe $\mathrm{Y}$, Matsuo K, Kubo M, Takahashi A, Ito H, et al. A genome-wide association study identifies two susceptibility loci for duodenal ulcer in the Japanese population. Nature genetics. 2012; 44: 430-4, S1-2.

24. Wang L, Sang Y, Tang J, Zhang RH, Luo D, Chen M, et al. Down-regulation of prostate stem cell antigen (PSCA) by Slug promotes metastasis in nasopharyngeal carcinoma. The Journal of pathology. 2015; 237: 411-22. 